\title{
Versión Española del Cuestionario de Función Reflexiva Parental (CFRP-18)
}

\author{
Spanish Version of the Parental Reflective Functioning Questionnaire (CFRP-18)
}

\author{
Leire Gordo $^{1}$, Leire Iriarte Elejalde ${ }^{2}$ y Ana Martínez-Pampliega ${ }^{3}$
}

\begin{abstract}
Resumen
Hasta la fecha, no existen instrumentos de autoinforme para evaluar la función reflexiva parental en lengua española. Por ello, el principal objetivo del presente estudio es analizar la estructura factorial y las propiedades psicométricas del Cuestionario de Función Reflexiva Parental (CFRP-18) en padres ( $\mathrm{n}=113)$ y madres $(n=433)$ de habla hispana de bebés entre 0 a 3 años. La media de edad de los padres fue 37.85 $(\mathrm{DT}=3.96)$ y la de las madres $36.15(\mathrm{DT}=4.19)$. Asimismo, se analiza la asociación entre las dimensiones del CFRP-18 y las características sociodemográficas de los progenitores. Como objetivo secundario, se analiza la invarianza factorial del CFRP-18 en madres y padres. El análisis factorial confirmatorio mostró un adecuado ajuste a los datos, aportando evidencia de la utilidad del CFRP-18 para evaluar el funcionamiento reflexivo parental. Este estudio aporta datos preliminares de la invariancia del CFRP-18.
\end{abstract}

Palabras clave: función reflexiva parental, instrumentos de evaluación, madres, padres

\begin{abstract}
To date, no self-report tool is available for assessing parental reflective functioning ability in Spanish. Therefore, the first aim of this study is to analyse the factor structure and the psychometric properties of the Parental Reflective Functioning Questionnaire (PRFQ) in Spanish-speaking fathers $(n=113)$ and mothers $(\mathrm{n}=433)$ of babies aged 0 to 3 years old. The average age of fathers was $37.85(\mathrm{SD}=3.96)$, and 36.15 years $(\mathrm{SD}=4.19)$ for mothers. In addition, the relationship of the dimensions of CFRP-18 with socio-demographic features of mothers and fathers is analyzed. Secondly, we investigate the factorial invariance of the CFRP-18 in mothers and fathers. A confirmatory factor analysis showed an adequate fit to the data, providing evidence that the CFRP-18 is a useful self-report tool to assess parental reflective functioning. This study also provides preliminary evidence for the factorial invariance of the CFRP-18.
\end{abstract}

Keywords: parental reflective functioning, assessment tools, mothers, fathers

\footnotetext{
${ }^{1}$ Doctora en Psicología. Profesora. Departamento de Psicología Social y del Desarrollo, Universidad de Deusto. Avenida de las Universidades, 24 (48007), Bilbao, España. Tel.: 944139 000. Ext: 2756. Correo: 1.gordo@ deusto.es

${ }^{2}$ Doctora en Psicología. Profesora, Universidad de Deusto. Departamento de Psicología Social y del Desarrollo. Universidad de Deusto. Avenida de las Universidades, 24 (48007), Bilbao, España. Tel.: 944139000 ext. 2756. Correo: 1.iriarte@ deusto.es

${ }^{3}$ Catedrática de Psicología. Profesora, Universidad de Deusto. Departamento de Psicología Social y del Desarrollo. Universidad de Deusto. Avenida de las Universidades, 24 (48007), Bilbao, España. Tel.: 944139000 ext. 2756. Correo: martinez.pampliega@ deusto.es

Revista Iberoamericana de Diagnóstico y Evaluación - e Avaliação Psicológica. RIDEP · №55 · Vol.2 · 5-17 · 2020

ISSN: 1135-3848 print /2183-6051online
} 


\section{Introducción}

La función reflexiva o mentalización alude a la capacidad de entender o interpretar las conductas de uno mismo y la de los otros en términos de estados mentales internos, como pueden ser los pensamientos, deseos y/o expectativas (Fonagy et al., 2016; Mitjavila, 2013).

Los estudios llevados a cabo hasta el momento sobre función reflexiva indican que se desarrolla en el contexto de las primeras relaciones de apego seguro del menor con sus cuidadores primarios y lo definen como un elemento clave para la autorregulación emocional y desarrollo del menor (Fonagy, Gergely, \& Target, 2007; Sharp \& Fonagy, 2008).

Debido a sus importantes implicaciones para el ajuste del menor, actualmente se ha puesto especial atención al concepto de función reflexiva en el campo de la parentalidad (Luyten, Mayes, Nijssens, \& Fonagy, 2017a). Y es que, como señalan Flores, Cortés y Góngora (2009), para poder entender la conducta de cada individuo, es fundamental conocer y entender el contexto en el que se encuentra inmerso y la naturaleza de las relaciones afectivas que el menor establece con sus cuidadores primarios.

La función reflexiva parental alude a la capacidad de los padres/madres de tratar de entender cómo los estados mentales afectan a la conducta de sus hijos/as lo cual hará que respondan de una manera más sensible y ajustada a las necesidades y deseos de sus hijos/as (Luyten et al., 2017a). Por ello, un padre/madre con un funcionamiento reflexivo adecuado en la relación con sus hijos/as tratará de reflexionar sobre las experiencias internas de sus hijo/as, será consciente de la opacidad de los estados mentales de sus hijos/as y de cómo éstos afectan a la conducta de sus hijos/as (Slade, 2005, 2008; Slade, Grunebaum, Huganir, \& Reeves, 2011). En definitiva, será capaz de "tener en mente la mente de su hijo/a" (Luyten, Nijssens, Fonagy, \& Mayes, 2017b; Mitjavila, 2013; Slade, 2005).

Teniendo en cuenta la importancia de la función reflexiva parental tanto para el ejercicio de la parentalidad como para el ajuste del menor, se han desarrollado diversos instrumentos de evaluación para tratar de captar el funcionamiento reflexivo de padres y madres. La mayoría de los instrumentos existentes se basan en entrevistas semiestructuradas: a) Parent Development Interview (PDI) revisada por (Slade, Aber, Bresgi, Berger, \& Kaplan, 2004); b) The Pregnancy Interview (PI) revisada por Slade et al. (2011); c) Adult Attachment Interview (AAI) (George, Kaplan, \& Main, 1985) y; d) Working Model of Child Interview (WMCI) (Zeanah, Benoit, \& Barton, 1986).

La limitación principal de este tipo de herramientas es la cantidad de tiempo que se necesita para su aplicación y el entrenamiento necesario para poder emplearlas, todo ello hace que sea muy difícil poder aplicar este tipo de instrumentos en estudios con muestras amplias y en contextos clínicos (Pajulo et al., 2018). Con el objetivo de poder superar esta limitación, uno de los objetivos de la investigación reciente ha sido crear instrumentos de autoinfome que evaluaran el funcionamiento reflexivo parental (Fonagy et al., 2016; Luyten, Fonagy, Lowyck, \& Vermote, 2012).

Para dar respuesta a este objetivo, Luyten et al. (2009) desarrollaron el Cuestionario de Función Reflexiva Parental (Parental Reflective Functioning Questionnaire, PRFQ-1). Se trata de una herramienta de autoinforme que consta de 39 ítems a través de los cuales se evalúa la función reflexiva en el contexto específico de las relaciones entre padres e hijos/as. Los autores originales del instrumento han identificado tres dimensiones clave de la función reflexiva parental, dos de estas dimensiones (Certeza en estados mentales (CEM) e Interés y curiosidad (IC) recogen aspectos positivos de la función reflexiva parental mientras que la Prementalización (PM) hace referencia a la tendencia de los padres/madres de utilizar estados no mentalizadores en la relación con sus hijos/as.

Posteriormente, con el objetivo de desarrollar una breve herramienta para evaluar el funcionamiento reflexivo parental, Luyten et al. (2017a) desarrollaron tres estudios que han aportado evidencia preliminar de la fiabilidad y validez del PRFQ como una breve medida multidimensional que consta de 18 ítems para evaluar la función reflexiva parental. En el primer estudio, se validó este instrumento en una muestra de 299 madres de bebés entre 0 a 3 años. Los resultados obtenidos tanto del análisis factorial exploratorio como del análisis factorial confirmatorio aportaron evidencia acerca de la 
estructura trifactorial del instrumento PRFQ (PM, IC y, CEM) El modelo propuesto obtuvo un buen ajuste a los datos $\left(\chi^{2}=217.73, \mathrm{df}=123, p<.001\right.$; $\chi^{2} / \mathrm{df}=1.77 ; \mathrm{RMSEA}=.05$ (CI .04-.06); CFI=.91). La consistencia interna $(\alpha)$ de cada una de las dimensiones fue adecuada (PM=.70; IC $=.75$; $\mathrm{CEM}=.82$ ) (Luyten et al., 2017a). En el segundo estudio, trataron de replicar estos resultados con madres $(\mathrm{N}=76)$ y padres $(\mathrm{N}=77)$ aportando evidencia de la invariancia factorial del PRFQ en función del género de los progenitores. En el estudio tres, se analizó la relación del PRFQ y el tipo de apego infantil medido a través de la técnica de la Situación extraña. Los resultados de estos estudios confirmaron la relación esperada entre el PRFQ y las dimensiones del apego parental, la disponibilidad emocional, estrés parental y el tipo de apego infantil (Luyten et al., 2017a).

Aunque el PRFQ ha sido traducido a varios idiomas, hasta la fecha, únicamente se han publicado los estudios de validación con una muestra de Italia (Pazzagli, Delvecchio, Raspa, Mazzeschi, \& Luyten, 2017) y Canadá (De Roo, Wong, Rempel, \& Fraser, 2019).

Debido a que el cuestionario de función reflexiva parental original (Parental Reflective Functioning Questionnaire, PRFQ) fue desarrollado y validado en inglés y no está disponible para padres/madres de habla hispana, el objetivo principal del presente estudio es traducir y validar el PRFQ en una muestra española. Como objetivo secundario, se pretende comprobar la invarianza factorial del Cuestionario de Función Reflexiva Parental (CFRP-18) (configural, métrica, escalar, estructural y residual) en función del género de los progenitores.

Se espera que la versión española del Cuestionario de Función Reflexiva Parental (CFRP18) replique la estructura tridimensional (PM, IC y, CEM) propuesta por los autores originales del instrumento, y que esta estructura sea invariante en función del género de los progenitores.

\section{Método}

\section{Participantes}

La muestra está compuesta por 433 madres y 113 padres, con bebés entre 0 y 3 años. La edad de las madres se ubicó en un rango de edad de 18 a 48 años y el rango de edad de los padres fue de
29 a 49 años. La media de edad de las madres $36.15(\mathrm{DT}=4.19)$ y la de los padres $37.85(\mathrm{DT}=$ 3.96) fue similar. La mayoría de los participantes tanto en el caso de las madres (95.8\%) como de los padres (96.3\%) fueron de nacionalidad española, y procedentes de familias biparentales (88\% de las madres y el $95.6 \%$ de los padres). El $68.5 \%$ de las madres y el $54.6 \%$ de los padres cuenta con estudios superiores y el $82 \%$ de las madres y el $93.7 \%$ de los padres están en activo laboralmente (Véase Tabla 1).

Tabla 1. Características sociodemográficas de la muestra en el estudio

\begin{tabular}{|c|c|c|}
\hline & $\begin{array}{l}\text { Madres } \\
(n=433)\end{array}$ & $\begin{array}{l}\text { Padres } \\
(\mathrm{n}=113)\end{array}$ \\
\hline Edad (años) $^{\mathrm{a}}$ & $36.15(4.19)$ & $37.85(3.96)$ \\
\hline $\begin{array}{l}\text { Nacionalidad española } \\
(\%)\end{array}$ & 95.8 & 96.3 \\
\hline \multicolumn{3}{|l|}{ Estado civil (\%): } \\
\hline Familias biparentales & 88 & 95.6 \\
\hline Solteros & 11.3 & 2.6 \\
\hline Separado/Divorciado & .5 & 1.8 \\
\hline Viudo/a & .2 & - \\
\hline \multicolumn{3}{|l|}{ Nivel de estudios (\%): } \\
\hline $\begin{array}{l}\text { Menos de estudios } \\
\text { primarios }\end{array}$ & .1 & .9 \\
\hline Estudios primarios & 3 & 2.7 \\
\hline Estudios secundarios & 25.2 & 39.1 \\
\hline $\begin{array}{l}\text { Estudios superiores } \\
\text { (universitarios, Máster y } \\
\text { doctorado) }\end{array}$ & 68.5 & 54.6 \\
\hline Otro & 3.2 & 2.7 \\
\hline \multicolumn{3}{|l|}{ Situación laboral (\%): } \\
\hline En activo & 82 & 93.7 \\
\hline En paro & 9.8 & 4.5 \\
\hline Baja laboral & 1.9 & 1.8 \\
\hline Sólo estudia & .9 & - \\
\hline Otra situación & 5.4 & - \\
\hline
\end{tabular}

\section{Instrumentos}

Cuestionario sociodemográfico. Fue construido ad hoc con el fin de obtener datos generales respecto a las características sociodemográficas de la muestra: edad, sexo, nacionalidad, estado civil, nivel de estudios y situación laboral.

PRFQ (Parental Reflective Functioning Questionnaire) (Luyten et al., 2017a). Como se ha señalado en la introducción, este instrumento persigue evaluar la función reflexiva parental de padres/madres de menores entre 0 a 5 años de edad a través de tres subescalas: Prementalización (PM), trata de identificar los estados parentales no mentalizadores "Mi hijo/a llora en presencia de 
desconocidos para avergonzarme"; Certeza en Estados Mentales (CEM), evalúa la identificación por parte de los padres/madres de la opacidad de los estados mentales de sus hijos/as "Siempre sé por qué mi hijo/a actúa de la forma en la que lo hace" e; Interés y Curiosidad (IC), incluye ítems relacionados con el interés genuino y/o curiosidad parental por los estados mentales de los hijos/as "Me pregunto muy a menudo sobre lo que mi hijo/a está pensando o sintiendo". Se responde en una escala tipo Likert que va de $1=$ "Muy en desacuerdo" a 7="Muy de acuerdo" (Luyten et al., 2017a).

En el presente estudio se empleó la versión española del Cuestionario de Función Reflexiva Parental (CFRP-18), el cual en su versión original demostró su solidez psicométrica. La versión española del CFRP-18 estará a disposición de los investigadores interesados bajo petición al autor de correspondencia del artículo.

\section{Procedimiento}

El desarrollo del presente estudio correlacional transversal fue apoyado por el Gobierno regional y aprobado por el Comité de Ética de Investigación de la Universidad de Deusto, antes de su puesta en marcha.

Para obtener la muestra (Octubre, 2017), se elaboró una carta que se envió a las familias a través de los/as educadores/as de escuelas infantiles de carácter público pertenecientes a la Comunidad Autónoma Vasca. En esta carta, se presentaban los objetivos del estudio y se facilitaban los datos de contacto de los investigadores responsables del estudio. De las 232 escuelas infantiles un $35 \%$ fueron seleccionadas aleatoriamente para ser visitadas, lo que supuso un total de 82 escuelas infantiles visitadas (34 en Vizcaya, 32 en Guipúzcoa y 16 en Álava). A través de los educadores/as de las escuelas infantiles se hacían llegar los cuestionarios a las familias. Aproximadamente, 15 días más tarde se recogían los cuestionarios cumplimentados por las familias en cada una de las escuelas infantiles.

En todo momento, se explicitó la voluntariedad de la participación y la confidencialidad de los datos. Asimismo, se informaba a los participantes que ante cualquier duda al cumplimentar los protocolos podían ponerse en contacto con los investigadores principales del estudio y que podían decidir dejar de participar en el estudio en el momento que lo desearan. No hubo remuneración económica por participar en el estudio.

\section{Estrategia de análisis}

En primer lugar, el cuestionario de función reflexiva parental original (PRFQ) fue adaptado al castellano, con el permiso de los autores del instrumento, mediante la metodología traducciónretrotraducción (Beaton, Bombardier, Guillemin, \& Ferraz, 2000). Para la adaptación de la versión española del PRFQ, en primer lugar dos personas españolas bilingües de origen británico realizaron una traducción independiente de los ítems originales del instrumento. En segundo lugar, se compararon ambas traducciones y se discutieron con el objetivo de alcanzar un consenso sobre la versión traducida. A continuación, una persona bilingüe española retrotradujo la versión española de nuevo al inglés. Finalmente, se compararon las dos versiones en inglés, retrotraducida y original, con el objetivo de comprobar la equivalencia conceptual.

A continuación, se analizó la distribución de los ítems del Cuestionario de Función Reflexiva Parental (CFRP-18) a través de los siguientes índices: media, desviación estándar, mediana, asimetría y curtosis. De cara a identificar aquellos ítems poco discriminativos, se consideraron los siguientes criterios: a) media más/menos una desviación típica de la media de la subescala; b) desviación típica menor a 0.5 ; c) valores de correlación con la subescala a la que pertenecen inferiores a $.40 \mathrm{y}$; d) aumento de la fiabilidad total de la subescala en más de 0.3 puntos con la eliminación del ítem (Morales, Urosa, \& Blanco, 2003; Streiner, Norman, \& Cairney, 2015).

Se calcularon los coeficientes de correlación entre cada ítem y la puntuación global de su dimensión correspondiente.

Se analizó la normalidad univariada a través de los índices de asimetría y curtosis, los cuales deben ser menores de 3 y 10 en valor absoluto respectivamente (Kline, 2005). La normalidad multivariante se analizó a través del coeficiente de Mardia. Según Bollen (1989) existe normalidad multivariada si el coeficiente de Mardia es inferior 
a $\mathrm{p}(\mathrm{p}+2)$, donde $\mathrm{p}$ es el número de variables observadas.

Respecto al objetivo principal del estudio, analizar la estructura tridimensional del Cuestionario de Función Reflexiva Parental (CFRP-18), se persiguió confirmar la estructura del cuestionario propuesta por los autores originales. Para ello, se utilizó el programa AMOS 25.0. y el ${ }^{1}$ método de máxima verosimilitud (ML) (Arbuckle, 2014). Se evaluó el nivel de bondad de ajuste de los datos al modelo de medida a través de la prueba de ji cuadrado de contraste de igualdad entre matrices $\left(\chi^{2}\right)$, que debería ser no significativa o mostrar valores bajos y la razón entre el $\chi^{2}$ y los grados de libertad del modelo $\left(\chi^{2} / \mathrm{gl}\right)$, que se recomienda que sea menor de 3 (Kline, 2005; Tabachnick \& Fidell, 2001). No obstante, el $\chi^{2}$ es muy sensible al tamaño de la muestra por lo que se utilizó también el error de aproximación cuadrático medio (Root Mean Square Error of Approximation - RMSEA) y su intervalo de confianza al $90 \%$, considerando aceptables valores entre .05 a .08 y muy buenos los valores menores que .05. Asimismo, se empleó el índice de bondad de ajuste (Goodness of Fit Index - GFI), el índice de ajuste comparativo (Comparative Fit Index - CFI), cuyos valores deben ser superiores a .90 (Bentler \& Bonett, 1980) y el índice estandarizado de residuo cuadrático medio (SRMR) cuyo valor debe ser menor a .08 para ser considerado aceptable (Fan \& Sivo, 2007). Para analizar la consistencia interna del Cuestionario de Función Reflexiva Parental (CFRP-18) se emplearon los coeficientes de fiabilidad Alpha de Cronbach $(\alpha)$ y la fiabilidad estimada mediante Omega de McDonald ( $\omega)$, estimador más preciso que alfa en ausencia de tauequivalencia o en presencia de ítems asimétricos (Trizano-Hermosilla \& Alvarado, 2016). Como valores de corte para la fiabilidad se consideraron los valores 50-.60 para las primeras fases de investigación, .80 para herramientas básicas de

\footnotetext{
${ }^{1}$ Uno de los revisores recomendó el uso de Máxima Verosimilitud Robusta, con el que se mejorarían los índices de bondad de ajuste. No obstante, ya que no existe una violación severa del supuesto de normalidad multivariante y no fue posible estimar la matriz de varianzas y covarianzas asintótica, se optó por el uso del método de estimación Máxima Verosimilitud (ML).
}

investigación y .90 para estudios con fines clínicos (Nunnally, 1967).

Finalmente, con el objetivo de analizar la invarianza del CFRP-18 en madres y padres, se llevó a cabo un análisis factorial confirmatorio multigrupo (AFCMG) con el programa AMOS 25.0. Este análisis se lleva a cabo a partir de una sucesión de modelos anidados jerárquicamente. El primer modelo (M1) sería el modelo base y permite analizar la invarianza configural, en este modelo no se establecen restricciones de igualdad en ninguno de los parámetros del modelo. En los siguientes modelos, se establecen restricciones de igualdad a diferentes parámetros entre los dos grupos. En el segundo modelo (M2), invarianza métrica, se fijaron las cargas factoriales de los ítems con el objetivo de garantizar que las cargas factoriales fueran equivalentes en los dos grupos. En el tercer modelo (M3), invarianza escalar, se añade como restricción la equivalencia a los valores interceptales. En el cuarto modelo (M4), invarianza estructural, las varianza y covarianzas de los factores se restringen a ser iguales en ambos grupos. Finalmente, en el quinto modelo (M5), invarianza residual, se añaden restricciones de igualdad a las varianzas y covarianzas del error. Para la interpretación de los resultados se tomó el valor de $\triangle \mathrm{CFI}$ y no las diferencias en Chi cuadrado ya que para ello, sería necesario implementar un método de estimación robusto. En concreto, valores inferiores a .01 de este indicador, fueron utilizados como criterio para poder determinar la invarianza entre modelos (Cheung \& Rensvold, 2002).

\section{Resultados}

En la Tabla 2, se observan los datos descriptivos y de discriminación de los ítems que componen cada una de las subescalas del CFRP18. Para la subdimensión Prementalización (PM) la media global fue de 1.56 con una desviación típica de .59 , lo que confiere un intervalo de selección entre los valores .97 a 2.15 excluyendo así, el ítem 16. En ningún caso la desviación típica ha sido inferior a 0.5 por lo que no se ha desestimado ningún ítem de la subescala por esta razón. Las puntuación media en PM de los padres fue de $1.70(\mathrm{DT}=.65)$ y de las madres 1.53 (DT=.57). Los datos indican que la distribuicíon de 
Tabla 2. Datos descriptivos y de discriminación de los ítems de las subescalas del CFRP-18

\begin{tabular}{|c|c|c|c|c|c|c|c|c|c|}
\hline Dimensión & Ítem & Rango & $\mathrm{M}$ & DT & $\mathrm{Me}$ & As & $\mathrm{Cu}$ & $\mathrm{r}$ & $\alpha$ \\
\hline \multirow{6}{*}{ PM } & 1 & \multirow{6}{*}{$1-7$} & 1.45 & .99 & 1 & 2.90 & 9.64 & $.57^{* *}$ & .55 \\
\hline & 4 & & 1.15 & .53 & 1 & 4.38 & 23.04 & & .56 \\
\hline & 7 & & 1.96 & 1.33 & 1 & 1.49 & 1.68 & $.62^{* *}$ & .57 \\
\hline & 10 & & 1.30 & .83 & 1 & 3.46 & 14.53 & $.55^{* * *}$ & .55 \\
\hline & 13 & & 1.30 & .82 & 1 & 3.55 & 14.92 & $.62^{* * *}$ & .52 \\
\hline & 16 & & 2.18 & 1.31 & 2 & .94 & .07 & $.64^{* * *}$ & .56 \\
\hline Total & & & 1.56 & .59 & 1.33 & 1.33 & 1.41 & --- & .60 \\
\hline \multirow{6}{*}{ CEM } & 2 & \multirow{6}{*}{$1-7$} & 3.85 & 1.49 & 4 & -.20 & -.72 & $.79^{* * *}$ & .70 \\
\hline & 5 & & 3.70 & 1.58 & 4 & -.24 & -.84 & $.73^{* * *}$ & .73 \\
\hline & 8 & & 3.48 & 1.49 & 3 & -.03 & -.82 & $.72^{* *}$ & .73 \\
\hline & 11 & & 3.87 & 1.59 & 4 & .36 & $\begin{array}{l}.02 \\
-.66\end{array}$ & $.59^{* * *}$ & .78 \\
\hline & 14 & & 4.54 & 1.50 & 5 & -.20 & -.63 & $.61^{* * *}$ & .77 \\
\hline & 17 & & 3.84 & 1.43 & 4 & -.13 & -.53 & $.68^{* * *}$ & .74 \\
\hline Total & & & 3.88 & 1.04 & 3.83 & .01 & -.52 & --- & .77 \\
\hline \multirow{6}{*}{ IC } & 3 & \multirow{6}{*}{$1-7$} & 5.84 & 1.30 & 6 & -1.40 & 2.19 & $.70^{* *}$ & .57 \\
\hline & 6 & & 5.36 & 1.37 & 6 & -.83 & .45 & $.68^{* * *}$ & .58 \\
\hline & 9 & & 6.14 & 1.15 & 6 & -1.83 & 4.35 & $.58^{* *}$ & .62 \\
\hline & 12 & & 5.83 & 1.12 & 6 & -1.18 & 2.28 & $.61^{* *}$ & .61 \\
\hline & 15 & & 5.63 & 1.37 & 6 & -1.32 & 1.77 & $.61^{* *}$ & .62 \\
\hline & 18 & & 6.12 & 1.41 & 7 & -1.86 & 3.09 & $.49^{* * *}$ & .677 \\
\hline Total & & & 5.82 & .78 & 5.83 & -.53 & .23 & --- & .66 \\
\hline
\end{tabular}

Nota. M: Media; DT: Desviación Típica; Me: Mediana; As: Asimetría; Cu: Curtosis; r: Correlación ítem con el resto de la escala; $\alpha=$ Alfa si se elimina el ítem; PM: Prementalización; CEM: Certeza en estados mentales; IC: Interés y curiosidad en estados mentales.

la dimensión PM presenta una asimetría positiva, por lo que existe una mayor aglomeración de las puntuaciones en el extremo superior. El ítem 4 de la dimensión PM es el que obtiene el valor más alto en la curtosis lo que indicaría una gran homogeneidad de los datos. Salvo tres ítems de la dimensión PM (4, 10 y 13), todos los ítems y la dimensión en su totalidad siguen una distribución normal. En cuanto a los coeficientes de correlación entre ítem y escala, todos los coeficientes se han situado por encima de .40. Por último, en ningún caso el valor que alcanzaría la fiabilidad de la escala si se retirase alguno de los ítems implicaría una mejora o empeoramiento en más de .3 puntos de la fiabilidad de la escala total.

Respecto a la dimensión Certeza en estados mentales (CEM), la media fue de 3.88 y la desviación típica de 1.04, todos los valores medios de los ítems correspondientes a esta dimensión se situaron dentro del intervalo de selección (2.84 a 4.92). La desviación típica de cada uno de los ítems de esta subescala en ningún caso se ha situado por debajo de .5 y todos los coeficientes de correlación entre ítem y escala se han situado por encima de .40. La puntuación media en CEM de los padres fue de 3.72 (DT=.94) y de las madres 3.92 (DT=1.06). La distribución de la dimensión CEM presenta una asimetría positiva por lo que existe una mayor aglomeración de puntuaciones en el extremo superior. Se obtiene un valor de curtosis negativo en esta dimensión. Todos los ítems de esta dimensión y la dimensión global siguen una distribución normal. En cuanto la fiabilidad de la escala, la eliminación del ítem 11 implicaría una mejora de la fiabilidad alcanzada por la escala total $(\alpha=.77)$. No obstante, esta mejora es inferior a .3 puntos.

Finalmente, atendiendo a la subescala Interés y curiosidad (IC), la media se situó en 5.82 y la desviación típica fue de .78. Todas las puntuaciones medias de los ítems se situaron dentro del intervalo de selección (5.04 a 6.6). Ninguna de las desviaciones típicas se situaron por debajo de .5. La puntuación media de los padres en IC fue de 5.61 (DT=.87) y de las madres de 5.87 (DT=.75). Finalmente, la distribución de la dimensión IC presenta una asimetría negativa indicando una mayor aglomeración de los datos en el extremo inferior. El valor de curtosis en esta dimensión es positivo y es el ítem 9 el que obtiene el valor más alto en la curtosis indicando una gran homogeneidad de los datos. Todos los ítems de esta dimensión y la dimensión en su totalidad siguen una distribución normal. Atendiendo a los coeficientes de correlación entre ítem y escala, todos se situaron por encima de .40. Respecto a la fiabilidad de la escala, los datos revelan una mejora 


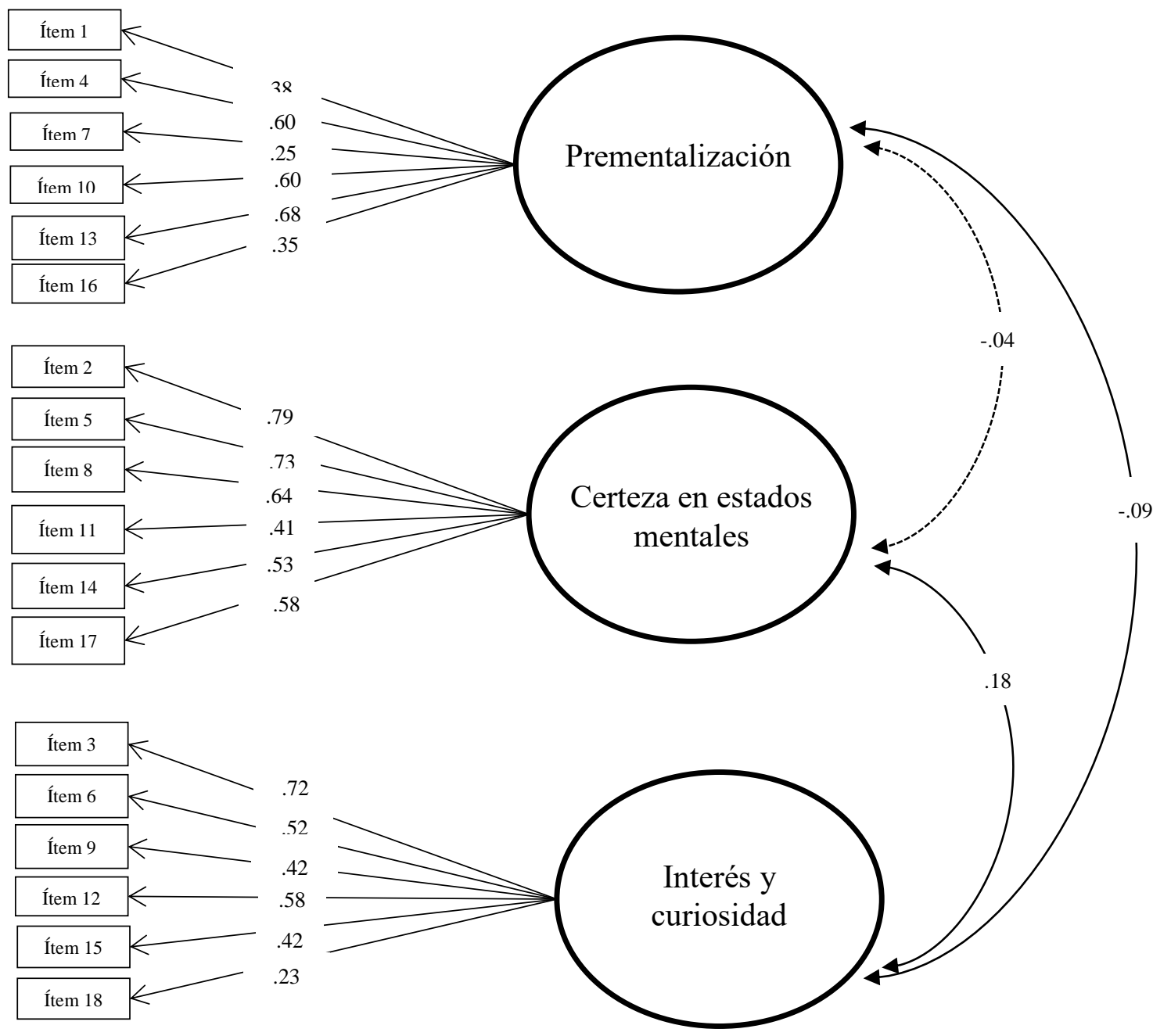

Figura 1. Análisis factorial confirmatorio del CFRP-18

de la fiabilidad alcanzada inferior a .3 puntos en la escala total $(\alpha=.66)$ si se elimina el ítem 18 (Véase Tabla 2).

Respecto a la normalidad multivariada, en el presente estudio son 18 las variables observadas y el coeficiente de Mardia provisto por el programa AMOS fue 151,502. Por tanto, se puede afirmar la existencia de normalidad multivariada ya que el valor aportado por el programa es menor que el producto $18(18+2)=360$.

A continuación, se llevó a cabo un análisis factorial confirmatorio (AFC) con las tres dimensiones del CFRP-18 propuestas por los autores originales del instrumento (PM, IC y, CEM). Inicialmente, el modelo no mostró un adecuado ajuste a los datos (CFI=.86). Los índices de modificación sugirieron añadir las covarianzas de los errores entre varios ítems, lo cual resultó en unos adecuados índices de ajuste al tomar cada uno de los índices analizados por separado: $\chi^{2}=296.983$, $\mathrm{gl}=127, p<.000, \chi^{2} / \mathrm{gl}=2.34 ; \mathrm{RMSEA}=.050(90 \%$ Intervalo de confianza [CI] [.042, .057]; CFI=.90, GFI=.94, SRMR=.06. Todos los ítems cargaron de forma significativa y en la dirección esperada en cada uno de sus factores correspondientes (Véase Figura 1). Los ítems 7, 16 y 18 fueron los que obtuvieron las cargas factoriales más bajas. El alpha de Cronbach $(\alpha)$ de las subescalas del Cuestionario de Función Reflexiva Parental (CFRP-18) fue adecuada: PM=.60, CEM=.77 e, IC $=.66$ y la fiabilidad Omega de McDonald $(\omega)$ se situó por encima del valor aceptable: $\mathrm{PM}=.66$, $\mathrm{CEM}=.78 \mathrm{e}, \mathrm{IC}=.67$.

Respecto a la asociación entre las dimensiones del CFRP-18 y la características sociodemográficas de la muestra (edad y nivel de estudios de progenitores, y edad de los hijos/as), únicamente se asociaron de forma significativa, y negativa, la dimensión CEM con la edad de los padres/madres $(r=-.12, p<.001)$ y la dimensión 
Tabla 3. Correlaciones entre las dimensiones del CFRP-18 y características sociodemográficas de la muestra

\begin{tabular}{lccc}
\hline & $\begin{array}{c}\text { Edad de los padres/ } \\
\text { madres }\end{array}$ & $\begin{array}{c}\text { Nivel de estudios de los } \\
\text { padres/madres }\end{array}$ & Edad hijos/as \\
\hline Prementalización & .05 & $-.09^{*}$ & .05 \\
Certeza en estados mentales & $-.12^{* *}$ & .02 & -.01 \\
Interés y curiosidad & -.06 & .02 & -.06 \\
\hline
\end{tabular}

Nota. ${ }^{*} p<.05 ;{ }^{* *} p<.001$

Tabla 4. Índices de ajuste global para la medición de invarianza del CFRP-18 en madres y padres

\begin{tabular}{lccccccc}
\hline & $\chi^{2}$ & Gl & P & CFI & $\Delta$ CFI & RMSEA & $\begin{array}{c}\text { RMSEA } \\
\text { IC-90\% }\end{array}$ \\
\hline Madres-Padres & & & & & & & \\
M1. Invarianza configural & 436.892 & 254 & .000 & .898 & - & .036 & $.031-.042$ \\
M2. Invarianza métrica & 466.469 & 269 & .000 & .890 & .008 & .037 & $.031-.042$ \\
M3. Invarianza escalar & 503.957 & 287 & .000 & .881 & .009 & .037 & $.032-.043$ \\
M4. Invarianza estructural & 523.776 & 293 & .000 & .872 & .009 & .038 & $.033-.043$ \\
M5. Invarianza residual & 562.593 & 315 & .000 & .863 & .009 & .038 & $.033-.043$ \\
\hline
\end{tabular}

Nota. $\chi 2=$ ji cuadrado; $\mathrm{gl}=$ grados de libertad; $\mathrm{p}=$ probabilidad; $\mathrm{CFI}=$ Índice de ajuste comparativo; $\Delta \mathrm{CFI}=$ Diferencia en valores del CFI; RMSEA=Error de aproximación cuadrático medio; RMSEA (Ic-90\%)=Intervalo de confianza RMSEA.

PM con el nivel de estudios de los padres/madres ( $r=-.09, p=.03$ ), (Véase Tabla 3).

Respecto al segundo objetivo del estudio, el análisis de la invarianza del CFRP-18 entre grupos (padres y madres), en la Tabla 4 se observa que el modelo base (M1) presentado muestra unos índices de ajuste aceptables, por lo que es posible asumir la invarianza configural entre padres y madres. Se observa que el descenso del CFI entre el M1 y el M2 ( $\triangle \mathrm{CFI}=.008)$ es menor que .01 , este dato permite asumir el criterio de invarianza métrica en ambos grupos. Asimismo, el descenso del CFI entre el M2 y M3 ( $\triangle \mathrm{CFI}=.009)$ es menor que .01 , lo que significa que se puede asumir el criterio de invarianza escalar en función del género de los progenitores. En el siguiente modelo (M4) se fijaron las varianzas y covarianzas de los factores $\mathrm{y}$, de nuevo, el descenso del CFI $(\Delta \mathrm{CFI}=.009)$ es menor que .01 , por lo que se asume el criterio de invarianza estructural. Finalmente, en el último modelo (M5) el descenso del CFI también es menor que .01 ( $\Delta \mathrm{CFI}=.009)$, por lo que se asume la invarianza residual.

\section{Discusión}

El presente estudio, dirigido a analizar la estructura, propiedades psicométricas e invarianza en función del género de los progenitores de la versión española del Cuestionario de Función Reflexiva Parental (CFRP-18), ha permitido confirmar la estructura tridimensional del instrumento CFRP-18 mostrando unas adecuadas propiedades psicométricas. Asimismo, se ha confirmado la invarianza factorial del instrumento en padres y madres. A continuación, se detallan los principales hallazgos del presente estudio.

En primer lugar, el hallazgo principal de este estudio es que los resultados del AFC confirman las tres dimensiones del CFRP-18 propuestas por los autores originales del instrumento (Prementalización -PM-, Certeza en Estados Mentales -CEM- e Interés y Curiosidad -IC-) (Luyten et al., 2017a). De esta forma, el instrumento ha confirmado la solidez de su estructura conceptual, pues como ya se encontró en otros estudios con muestras de madres y padres italianos y canadienses, nuestros resultados han vuelto a replicar la estructura tridimensional original del cuestionario (De Roo et al., 2019; Pazzagli et al., 2017).

En cuanto a las propiedades psicométricas de la versión española del CFRP-18, los datos son similares a lo encontrado en el estudio con padres/madres italianos en el que el coeficiente de Alpha de Cronbach $(\alpha)$ osciló entre .61 y .78 (Pazzagli et al., 2017). En cambio, la consistencia interna $(\alpha)$ de cada una de las dimensiones fue mayor en el estudio original que en el presente estudio (PM=.70; IC=.74; CEM=. 82) (Luyten et al., 2017a). Fue la versión canadiense la que obtuvo mayores índices de fiabilidad en cada una de las dimensiones (Alpha de Cronbach $(\alpha)$ osciló entre .88 y .91) al eliminar los ítems 11 y 18 (De Roo et al., 2019).

En la versión española, al igual que en la versión original (Luyten et al., 2017a), es la 
dimensión PM la que obtuvo una menor fiabilidad $(\alpha)$ en comparación con el resto de dimensiones. Al igual que lo que se observa en la versión canadiense (De Roo et al., 2019), es importante señalar que si se elimina el ítem 11 de la dimensión CEM y el ítem 18 de la dimensión IC mejoraría la fiabilidad total de su dimensión correspondiente. No obstante, en el presente estudio se ha optado por mantener la integridad de la escala con el fin de favorecer comparaciones internacionales.

Se analizó también la correlación de cada ítem con su dimensión correspondiente, y los coeficientes de correlación se situaron entre $r=$ .51 y $r=.79$, lo que indica una adecuada homogeneidad de los ítems (Briggs \& Cheek, 1986). En cuanto a las cargas factoriales, todos los ítems se situaron por encima de .30 (Bandalos \& Finney, 2010; Guadagnoli \& Velicer, 1988; McDonald, 1985) menos los ítems 7 ("Me resulta difícil participar activamente en juegos simbólicos/imaginarios con mi hijo/a") y 18 ("Creo que intentar adivinar qué siente mi hijo/a no tiene sentido"). No obstante, estos datos son coherentes con los obtenidos por Luyten et al. (2017a) en su estudio de validación del PRFQ, donde también encontraron que los ítems 7 y 18 obtuvieron cargas factoriales por debajo de $.30 \mathrm{al}$ analizar la invarianza estructural del instrumento en función del género de los progenitores (Luyten et al., 2017a). Asimismo, en los estudios desarrollados con muestra canadiense e italiana, los autores también indicaron que el ítem 18 no funcionaba adecuadamente (De Roo et al., 2019; Pazzagli et al., 2017). Según Deroo et al., (2018), esto puede ser explicado por la mezcla de una formulación tanto negativa como positiva en un mismo ítem ya que puede dar lugar a dificultades a la hora de entender y responder estos ítems. Una posible alternativa para mejorar los índices psicométrica del CFRP-18 podría ser modificar el fraseo de los reactivos o eliminarlos. Sin embargo, como se ha indicado previamente, en el presente estudio se ha optado por mantener la escala completa propuesta por los autores por ser fieles a la escala original y favorecer la comparabilidad de resultados.

Las correlaciones interfactor obtenidas en el presente estudio fueron bajas. En el estudio original del instrumento la dimensión PM no correlacionó con las dimensiones CEM e IC. Sin embargo, se observó una asociación significativa entre las dimensiones CEM e IC (Luyten et al., 2017a). Son necesarios más estudios empleando el CFRP-18 con el objetivo de seguir explorando su estructura y propiedades psicométricas.

Atendiendo a las puntuaciones medias obtenidas en el presente estudio en las dimensiones del CFRP-18, se observa que las puntuaciones en la dimensión IC se sitúan por encima de la media, tanto en la muestra total como diferenciando a los padres de las madres, indicando que los progenitores participantes en este estudio se caracterizan por mostrar un interés y curiosidad por los estados mentales de sus hijos/as. No obstante, es importante señalar que las puntuaciones en esta dimensión no son altas. Esto es acorde con lo señalado por Luyten et al. (2017a) al señalar que puntuaciones medias en esta dimensión son adaptativas mientras que puntuaciones muy bajas o muy altas no son adaptativas. De hecho, en su estudio encontraron que el IC y la CEM se asociaron con la disponibilidad emocional de los padres/madres, pero también puntuaciones altas en estas dimensiones se asociaban con la intrusividad materna. Las puntuaciones medias en CEM en el presente estudio se situaron por debajo de la media tanto en la muestra total como diferenciando a los padres de las madres. En cuanto a las puntuaciones medias en la dimensión PM en el presente estudio éstas se situaron por debajo de la media tanto en la muestra total como en los padres y en las madres, indicando así un menor uso de modos prementalizadores en la relación con sus hijos/as. Esto puede explicarse por las características de los participantes que participaron en el estudio, puesto que en otros estudios desarrollados con progenitores con altos niveles de estrés parental se ha observado un mayor uso de modos prementalizadores en la relación con sus hijos/as (Fonagy \& Luyten, 2009; Nijssens, Bleys, Casalin, Vliegen, \& Luyten, 2018).

En cuanto a la relación entre las características sociodemográficas de la muestra y las dimensiones del CFRP-18, la dimensión IC no se asoció con ninguna de las características sociodemográficas de la muestra. Por el contrario, la asociación entre la dimensión PM con el nivel 
de estudios de los padres/madres aunque fue pequeña, fue negativa y significativa. Lo encontrado indica que a menor PM mayor es el nivel de estudios de los padres/madres. Estos resultados, acordes con lo encontrado por Luyten et al. (2017a), parecen apoyar el hecho de que padres/madres con un nivel educativo inferior pueden mostrar mayores dificultades de introspección y de poder mentalizarse a uno mismo y al otro, por lo que pueden mostrar una tendencia a percibir una intencionalidad negativa detrás de la conducta de los otros y por consiguiente, mostrar fallas en su capacidad mentalizadora (Fonagy, Luyten, \& Allison, 2015). La dimensión CEM, por su parte, mostró una asociación pequeña y negativa con la edad de los progenitores. Esto es acorde con lo encontrado por Rutherford, Booth, Luyten, Bridgett, \& Mayes (2015), sugiriendo que a mayor edad, menor es la habilidad de padres/madres de reconocer la opacidad de los estados mentales de sus hijos/as.

La baja asociación entre las dimensiones del CFRP-18 y las característica sociodemográficas de la muestra puede ser explicada por la homogeneidad de la muestra, al igual que lo encontrado por Luyten et al. (2017a) en los tres estudios desarrollados para la validación original del PRFQ. Es decir, parece respaldarse que cuanto más homogéneas son las muestras y cuanto mejor funcionamiento parental muestran los padres/madres, las relaciones entre las dimensiones del CFRP-18 y las variables sociodemográficas son más modestas.

Respecto al segundo objetivo del estudio, al igual que en el estudio llevado a cabo por los autores originales del instrumento (Luyten et al., 2017a), los resultados permiten asumir el criterio de invarianza factorial en función del género de los progenitores. En primer lugar, se confirma la invarianza configural, lo que señala que los padres/madres conceptualizan de la misma manera el constructo de función reflexiva parental (Cheung \& Rensvold, 2002). En segundo lugar, también se observa invarianza métrica entre madres y padres para el Cuestionario de Función Reflexiva Parental (CFRP-18), es decir, la fuerza de la relación entre los ítems y el constructo al que pertenecen es igual, esto supone que las madres y los padres interpretan los ítems de las escalas del CFRP-18 de la misma manera. En tercer lugar, se confirma la invarianza escalar. Este tipo de invarianza supone que las medidas de las escalas se definen operativamente de la misma manera en madres y en padres (Gregorich, 2006). En cuarto lugar, se observa invarianza estructural por lo que la relación entre los constructos (por ejemplo, covarianzas) son iguales entre grupos (Cheung \& Rensvold, 2002). Y finalmente, se confirma la invarianza residual, es decir, existe consistencia entre los grupos a la hora de responder a los ítems. Esto implica que ambos progenitores reaccionan de forma similar ante la escala, debido a que el vocabulario y las expresiones implicadas en el instrumento son igualmente comprensibles para ambos (Cheung \& Rensvold, 2002; Malpass, 1977; Mullen, 1995). Debido a la creciente atención que el constructo de función reflexiva está recibiendo en el campo de la parentalidad, es fundamental contar con herramientas de medición que sean válidas y confiables y que, como el CFRP-18, sean invariantes en función del género de los progenitores. Únicamente de esta manera, se llevará a cabo una adecuada evaluación tanto de la figura materna como de la paterna, lo que nos permitirá comparar sus puntuaciones medias, $\mathrm{y}$ así seguir explorando diferencias de género y poder obtener conclusiones válidas.

Este estudio presenta varias limitaciones: en primer lugar, el CFRP-18 es un instrumento de autoinforme por lo que la información que se obtiene es limitada si la comparamos con las entrevistas semiestructuradas diseñadas para evaluar la función reflexiva parental. No obstante, es una herramienta breve de screening que puede ser administrada fácilmente en muestras amplias y en aquellos contextos clínicos en los que la evaluación de los procesos, a pesar de ser relevante, es secundaria a las prioridades de la intervención. Una segunda limitación de este estudio es la homogeneidad de la muestra, ya que la mayoría de padres/madres tenían un nivel socioeconómico y educacional medio-alto. Para futuros estudios sería importante la utilización del CFRP-18 en población clínica y de riesgo y generalizar los resultados obtenidos. En tercer y último lugar, la participación de los padres en el estudio es menor que la de las madres. Esta diferencia está vinculada a los estereotipos socialmente establecidos relacionados con la maternidad (Mota, Calleja, Sánchez, \& Carreño, 
2019) y a la todavía implicación diferencial de los progenitores en temas familiares. No obstante, es importante propiciar la participación de los padres y estudiar posibles diferencias de género en la función reflexiva parental.

En conclusión, el presente estudio aporta evidencia preliminar de la fiabilidad y validez de la versión española del Cuestionario de Función Reflexiva Parental (CFRP-18) y subraya la utilidad del CFRP-18 como una breve herramienta de autoinforme fácil y rápida de administrar para evaluar la función reflexiva parental.

\section{Referencias}

Arbuckle, J. L. (2014). Amos (Version 23.0) [Computer Software]. Chicago, IL: IBM SPSS.

Bandalos, D. L., \& Finney, S. J. (2010). Factor analysis: Exploratory and confirmatory. In G. R. Hancock \& R. O. Muelle (Eds.), The reviewer's guide to quantitative methods in the Social Science (pp. 93-114). New York: Routledge.

Beaton, D. E., Bombardier, C., Guillemin, F., \& Ferraz, M. B. (2000). Guidelines for the process of cross-cultural adaptation of selfreport measures. Spine, 25(24), 3186-3191. https://doi.org/10.1097/00007632-20001215000014

Bentler, P. M., \& Bonett, D. G. (1980). Significance tests and goodness of fit in the analysis of covariance structures. Psychological Bulletin, 88(3), 588-606. https://doi.org/10.1037/0033-2909.88.3.588

Bollen, K. A. (1989). Structural equations with latent variables. Hoboken, NJ: John Wiley \& Sons, Inc.

Briggs, S. R., \& Cheek, J. M. (1986). The role of factor analysis in the development and evaluation of personality scales. Journal of Personality, 106-148. https://doi.org/10.1111/j.14676494.1986.tb00391.x

Cheung, G. W., \& Rensvold, R. B. (2002). Evaluating goodness-of-fit indexes for testing measurement invariance. Structural Equation Modeling: A Multidisciplinary Journal, 9(2), 233-255.
https://doi.org/10.1207/S15328007SEM0902_5

De Roo, M., Wong, G., Rempel, G. R., \& Fraser, S. N. (2019). Advancing optimal development in children: Examining the construct validity of a Parent Reflective Functioning Questionnaire. JMIR Pediatrics and Parenting, $\quad 2(1), \quad$ e11561. https://doi.org/10.2196/11561

Fan, X., \& Sivo, S. A. (2007). Sensitivity of fit indices to model misspecification and model types. Multivariate Behavioral Research, 42(3), 509-529. https://doi.org/10.1080/00273170701382864

Flores, G. M., Cortés, A. M., \& Góngora, C. E. (2009). Desarrollo y validación de la escala de percepción de prácticas parentales de crianza para niños en una muestra mexicana. Revista Iberoamericana de Diagnóstico y Evaluación - e Avaliação Psicológica, 28(2), 45-66.

Fonagy, P., Gergely, G., \& Target, M. (2007). The parent-infant dyad and the construction of the subjective self. Journal of Child Psychology and Psychiatry, 48(3-4), 288-328. https://doi.org/10.1111/j.1469-

7610.2007.01727.x

Fonagy, P., \& Luyten, P. (2009). A developmental, mentalization-based approach to the understanding and treatment of borderline personality disorder. Development and Psychopathology, 21(4), 1355-1381. https://doi.org/10.1017/S0954579409990198

Fonagy, P., Luyten, P., \& Allison, E. (2015). Epistemic petrification and the restoration of epistemic trust: A new conceptualization of borderline personality disorder and its psychosocial treatment. Journal of Personality Disorders, 29(5), 575-609. https://doi.org/10.1521/pedi.2015.29.5.575

Fonagy, P., Luyten, P., Moulton-Perkins, A., Lee, Y. W., Warren, F., Howard, S., ... Lowyck, B. (2016). Development and validation of a self-report measure of mentalizing: The Reflective Functioning Questionnaire. PLoS ONE, 11(7), e0158678. https://doi.org/10.1371/journal.pone.0158678 George, C., Kaplan, N., \& Main, M. (1985). The Adult Attachment Interview. Unpublished manuscript, Berkeley, CA: University of California.

Gregorich, S. E. (2006). Do self-report 
instruments allow meaningful comparisons across diverse population groups? Medical Care, 44(Suppl 3), S78-S94. https://doi.org/10.1097/01.mlr.0000245454.12 $228.8 \mathrm{f}$

Guadagnoli, E., \& Velicer, W. F. (1988). Relation of sample size to the stability of component patterns. Psychological Bulletin, 103(2), 265275.

https://doi.org/10.1037/0033-2909.103.2.265

Kline, R. B. (2005). Principles and practice of structural equation modeling (2nd ed). New York, NY: The Guilford Press.

Luyten, P., Fonagy, P., Lowyck, B., \& Vermote, R. (2012). Assessment of mentalization. In A. W. Bateman \& P. Fonagy (Eds.), Handbook of Mentalizing in Mental Health Practice (pp. 43-66). Arlington, VA: American Psychiatric Publishing.

Luyten, P., Mayes, L. C., Nijssens, L., \& Fonagy, P. (2017). The parental reflective functioning questionnaire: Development and preliminary validation. PLOS ONE, 12(5), e0176218. https://doi.org/10.1371/journal.pone.0176218

Luyten, P., Mayes, L., Sadler, L., Fonagy, P., Nicholls, S., Crowley, M., \& Slade, A. (2009). The Parental Reflective Functioning Questionnaire-1 (PRFQ-1). Unpublished manual, Leuven, Belgium: University of Leuven.

Luyten, P., Nijssens, L., Fonagy, P., \& Mayes, L. C. (2017). Parental reflective functioning: theory, research, and clinical applications. The Psychoanalytic Study of the Child, 70(1), 174199.

https://doi.org/10.1080/00797308.2016.1277901

Malpass, R. S. (1977). Theory and method in cross-cultural psychology. American Psychologist, 32(12), 1069-1079. https://doi.org/10.1037/0003-066X.32.12.1069

McDonald, R. P. (1985). Factor analysis and related methods. Hillsdale, NJ: LEA.

Mitjavila, M. (2013). Investigación y aportaciones de Peter Fonagy: Una revisión desde el 2002 al 2012. Temas de Psicoanálisis, 5, 1-17. Retrieved from http://www.temasdepsicoanalisis.org/wpcontent/uploads/2013/01/PDF-

MITJAVILA1.pdf

Morales, P., Urosa, B., \& Blanco, A. (2003).
Construcción de escalas de actitudes tipo Likert: Una guía práctica. Madrid: La Muralla.

Mota, C., Calleja, N., Sánchez, C., \& Carreño, J. (2019). Escala de Creencias sobre la Maternidad: Construcción y validación en mujeres mexicanas. Revista Iberoamericana de Diagnóstico y Evaluación - e Avaliação Psicológica, 50(1), 163-172. https://doi.org/10.21865/RIDEP50.1.13

Mullen, M. R. (1995). Diagnosing measurement equivalence in cross-national research. Journal of International Business Studies, 26(3), 573-596. https://doi.org/10.1057/palgrave.jibs.8490187

Nijssens, L., Bleys, D., Casalin, S., Vliegen, N., \& Luyten, P. (2018). Parental attachment dimensions and parenting stress: The mediating role of parental reflective functioning. Journal of Child and Family Studies, 27(6), 2025-2036. https://doi.org/10.1007/s10826-018-1029-0

Nunnally, J. C. (1967). Psychometric theory. NY: McGraw-Hill.

Pajulo, M., Tolvanen, M., Pyykkönen, N., Karlsson, L., Mayes, L., \& Karlsson, H. (2018). Exploring parental mentalization in postnatal phase with a self-report questionnaire (PRFQ): Factor structure, gender differences and association with sociodemographic factors. The Finn Brain Birth Cohort Study. Psychiatry Research, 262, 431-439.

https://doi.org/10.1016/j.psychres.2017.09.020

Pazzagli, C., Delvecchio, E., Raspa, V., Mazzeschi, C., \& Luyten, P. (2017). The Parental Reflective Functioning Questionnaire in mothers and fathers of school-aged children. Journal of Child and Family Studies, 27(1), 80-90.

https://doi.org/10.1007/s10826-017-0856-8

Rutherford, H. J., Booth, C. R., Luyten, P., Bridgett, D. J., \& Mayes, L. C. (2015). Investigating the association between parental reflective functioning and distress tolerance in motherhood. Infant Behavior and Development, $\quad 40, \quad 54-63$. https://doi.org/10.1016/j.infbeh.2015.04.005

Sharp, C., \& Fonagy, P. (2008). The parent's capacity to treat the child as a psychological 
agent: Constructs, measures and implications for developmental psychopathology. Social Development, 17(3), 737-754. https://doi.org/10.1111/j.1467-507.2007.00457.x

Slade, A. (2005). Parental reflective functioning: An introduction. Attachment and Human Development, $\quad 7(3), \quad 269-281$. https://doi.org/10.1080/14616730500245906

Slade, A. (2008). Working with parents in child psychotherapy: Engaging the reflective function. In F. N. Busch (Ed.), Psychoanalytic Inquiry book series. Mentalization: Theoretical considerations, research findings, and clinical implications (pp. 207-234). Mahwah, NJ: Analytic Press.

Slade, A., Aber, J. L., Bresgi, I., Berger, B., \& Kaplan, M. (2004). The parent development interview revised. Unpublished protocol, New York, NY: The City University of New York.

Slade, A., Grunebaum, L., Huganir, L., \& Reeves, M. (2011). The pregnancy interview, revised. New York: The Psychological Center, The City College of New York.

Streiner, D. L., Norman, G. R., \& Cairney, J. (2015). Health measurement scales: A practical guide to their development and use (5 ed). Nueva York: Oxford University Press.

Tabachnick, B. G., \& Fidell, L. S. (2001). Using multivariate statistics (4th ed.). Needham, MA: Allyn \& Bacon.

Trizano-Hermosilla, I., \& Alvarado, J. M. (2016). Best alternatives to Cronbach's alpha reliability in realistic conditions: Congeneric and asymmetrical measurements. Frontiers in $\begin{array}{lll}\text { Psychology, } & 769 .\end{array}$ https://doi.org/10.3389/fpsyg.2016.00769

Zeanah, C. H., Benoit, D., \& Barton, M. (1986). Working model of the child interview. Unpublished Manuscript. 